\title{
Design of High Precision Lighting Control System for Machine Vision Image Acquisition
}

\author{
Kai-Peng YU *, Ji-Ye HUANG, Shuang WU \\ College of Electron Information, HangZhou Dianzi University, Hangzhou, China \\ yukaipeng123@163.com
}

\begin{abstract}
Keywords: LED lighting control; Machine vision; STM32; CAN bus;
Abstract. In this paper, a high precision lighting control system based on TPS92640 is proposed, which meet the requirements of image acquisition in light source. The whole system adopts modular design, including STM32 master module, human-computer interaction interface, light-driven module based on TPS92640, 485 communication module, CAN bus communication module, AD isolation voltage acquisition module. Each module is interrelated to achieve the adjustment of the light intelligently. The lighting strips consist of dense and ordered LED permutations. In theory, the control accuracy of the light drive module can reach $1 / 2000$, but because of the project requirements we actually apply the accuracy of 1/100. After testing, the light intensity of the system is strong and uniform. It can greatly improve the quality of the image acquisition and conducive to follow-up image processing.
\end{abstract}

\section{Introduction}

With the rapid development of industrial control in our country, machine vision is also increasingly being applied to the industrial field. How to provide a high quality lighting control system is very important for image acquisition. Most of the traditional lighting control system have obvious shortcomings. For example, the brightness of some lighting control systems is constant, which can't be adjusted with environmental factors [1]; As another example ,the brightness of some light control system is adjustable, but the adjustable level is very limited, that can't meet some high-precision machine vision project [2]; There are also some lighting control systems without human computer interface, which's inconvenient for people to control [3-4]. LED lighting is the trend of development: on the one hand, it is due to the advantages of LED itself; on the other hand, the LED industry is in line with the current "green, low-carbon life" [5]. The lighting control system completed in this paper can solve all the above mentioned problems, which can improve the quality of the light source and the image collecting environment of the camera, enhance the accuracy of subsequent image processing. At the same time, the lighting control system has a self-test function, and it can feedback the wrong message to the human-computer interaction interface [6].

\section{Composition of Lighting Control System}

A complete lighting control system for machine vision image acquisition is mainly composed of STM32 master module, human-computer interface, light-driven module based on TPS92640, 485 communication module, CAN bus communication module, and AD isolation voltage acquisition module. Each module through different modes of communication to get together as shown in Fig.1. The human-computer interaction interface communicates with the master board via 485 bus. It is very flexible to control 11 road lighting module corresponding to adjust the brightness of the lights through the human-computer interaction interface, and at the same time, the main control terminal can monitor the working condition of each road, where the lights work is not normal, the host will feedback the wrong message to the human-computer interaction interface. The master board and four cameras connected with each other through the CAN bus, each camera can feedback the brightness information to the master board based on its own ID, and then transmits the information to the human-computer interaction interface from the master board. 


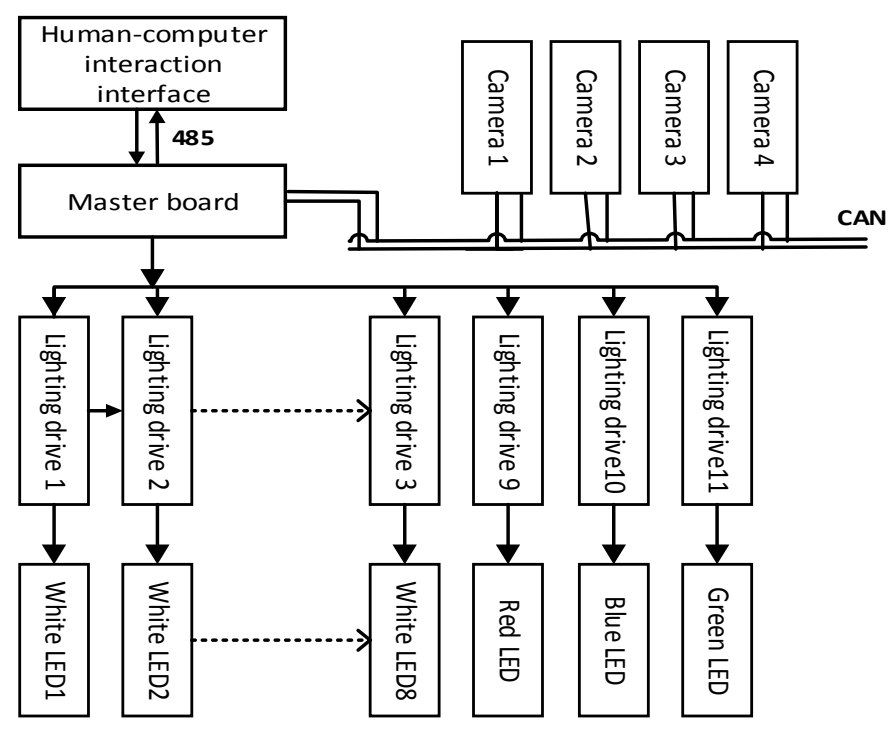

Fig.1 Light control system topology

\section{Hardware Design}

\subsection{Controller hardware design}

The main controller of the lighting control system uses STMicroelectronics STM32F407ZGT6, it is used in large capacity ARM Cortex-M4 kernel enhanced 32-bit microcontrollers and the highest frequency can reach $168 \mathrm{MHz}$. This processor is equipped with a wealth of peripheral resources, the communication interface of commonly used industrial sectors are reserved, such as CAN, USART, SPI and so on.

\subsection{High precision LED driver circuit}

High-precision light-driven circuit is designed based on TPS92640, which supports analog voltage and PWM wave in two ways to adjust the brightness of LED lights. We use the PWM wave drive mode, and this mode is more intelligent adjustment, you can easily control the brightness of the LED through the human-computer interface. The drive circuit design is shown in Fig.2, the VIN is connected to the $12 \mathrm{~V}$ switching power supply, used to power the LED. PWM is connected to the microcontroller PWM output IO port, that different duty cycle PWM waves can control the different brightness of the LED, here the frequency $T_{P W M}$ of our PWM is set to 10khz. According to the duty cycle of the PWM wave, the chip coordinates the on-off of Q1 and Q2 (here we use NTD3055-150 for Q1 and Q2) to achieve the regulation of the current flowing through the LED size, and the inductance L1 makes the current more smooth close to the DC. IADJ is the analog voltage control terminal, here we pass on the resistor divider of reference voltage $V_{R E F}$ (3V constant) to give IADJ a fixed voltage value $V_{I A D J}$, and the maximum effective current of LED $I_{M L E D}$ (that is, when the duty cycle of $100 \%$ ) and $V_{I A D J}$ are in relationship as formula (1). The actual flow through the LED current $I_{L E D}$ is related to the PWM duty cycle, and the relationship between the formula (2), where the duty cycle is $D_{D I M}$. The switching frequency $f_{s w}$ relation of the current oscillation in the circuit is shown as (3), and the range of $f_{s w}$ is from $10 \mathrm{Khz}$ to $1 \mathrm{Mhz}$. If you want to get a lower ripple current in this circuit, you require a larger inductance and a higher switching frequency. So here we will set the switching frequency to $1 \mathrm{Mhz}$, that is, the switching cycle $\mathrm{T}$ is 1us. As shown in Fig.3, $T_{P W M}$ is the period of the PWM, the switch of NFET is synchronized at the high level of PWM, and the low level segment of the PWM is disabled, in the Fig, the $I_{M L E D}$ is the maximum effective current and $I_{L E D}$ is the actual current at that duty cycle. Corresponding to Fig. 2, $R_{C S}$ is $R_{11}, R_{\text {VOUT } 1}$ is $R_{12}, R_{\text {VOUT } 2}$ is $R_{6}, R_{\text {on }}$ is $R_{2}$, and $C_{O N}$ is $C_{5}$. 


$$
\begin{aligned}
& I_{M L E D}=\frac{\frac{V_{I A D J}}{10}}{R_{C S}} \\
& I_{L E D}=D_{D I M} \times I_{M L E D} \\
& f_{S W}=\frac{1}{T}=\frac{R_{V O U T 1}+R_{V O U T 2}}{R_{V O U T 2}} \times \frac{1}{R_{O N} \times C_{O N}}
\end{aligned}
$$

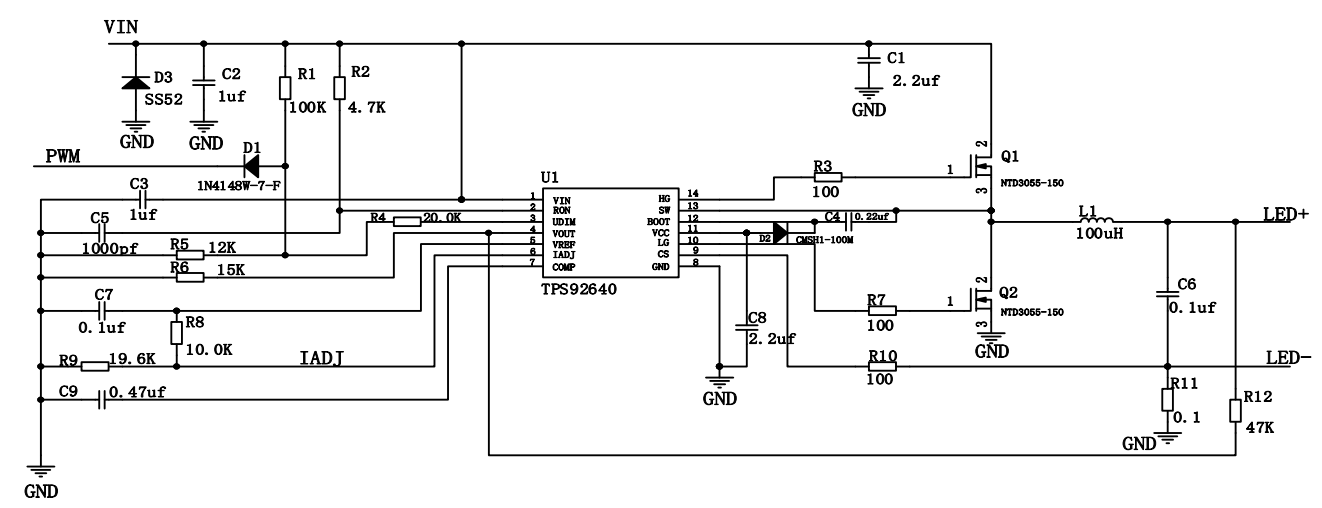

Fig.2 High precision light driving circuit

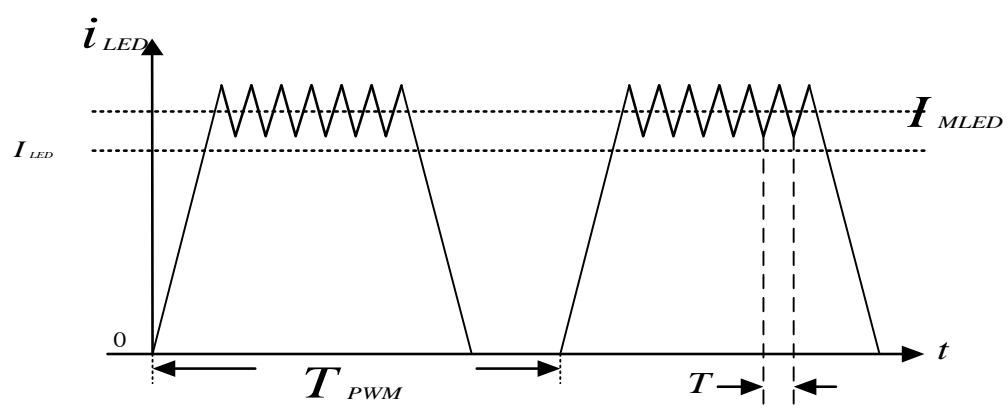

Fig.3 The actual current waveform through the LED

\subsection{5 communication circuit}

485 communication has many advantages. For example, its transmission distance is very far and the transmission signal is not susceptible to external interference, so it can be a good way to establish a MCU and PC remote communication. It is proved by test that the 485 communication circuit which we designed as Fig.4 is stable and safe in practical application.

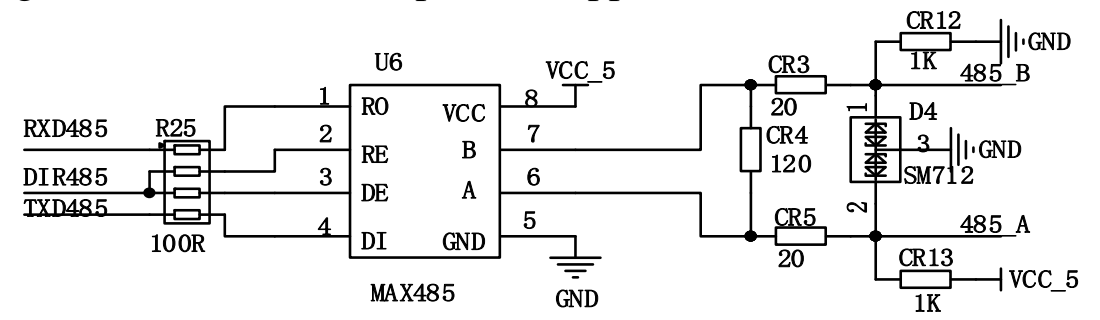

Fig.4 485 communication circuit

\subsection{CAN communication circuit}

CAN bus is an international standard industrial field bus. In addition, a CAN bus has more nodes, and the communication protocol comes with CRC check. Compared with 485, each node of CAN has a CAN controller, when multiple nodes send signals simultaneously, the communication sequence can be arbitrated in accordance with ID, therefore, there will be no communication 
confusion. Because in our lighting control system, a MCU will communicate with multiple cameras simultaneously, so the CAN bus is chosen to link them together. Fig.5 is a CAN drive circuit.. It is proved by test that the drive circuit is stable and reliable in practical applications, and the communication between the cameras and the main board is efficient and orderly.

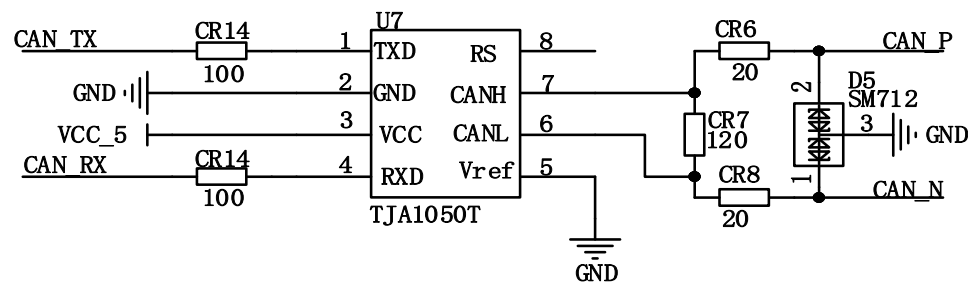

Fig.5 CAN communication circuit

\subsection{Isolation AD voltage acquisition circuit}

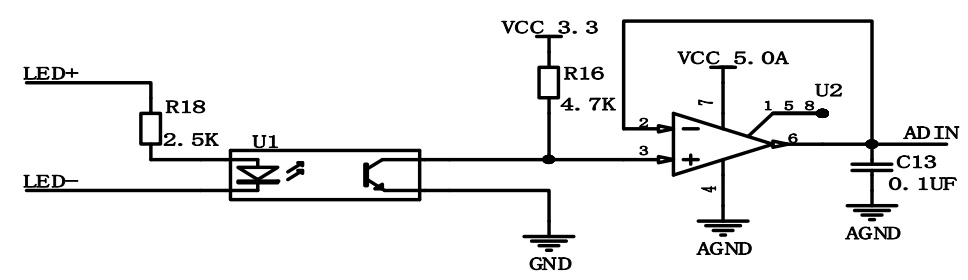

Fig.6 Isolation $\mathrm{AD}$ voltage acquisition circuit

The circuit of Fig.6 is used to collect the voltage of the LED. To protect the MCU from the abnormal current at the test end, we use the optocoupler isolation design, and to improve the quality of the voltage acquisition, we added a follower between the optocoupler and the AD. Table 4 shows the actual duty cycle of the PWM and the corresponding LED voltage, which collected by this circuit. Through this data, we can make a simple judgment, when we have given the PWM, the corresponding LED is in a normal working state or no.

\subsection{Theoretical analysis of RGB tricolor light plate}

The background setting is critical in image acquisition. For collecting different objects, the appropriate background color will greatly improve the quality of image acquisition. Here we use the RGB lighting board as the background, so you can adjust the background flexibly .The RGB each has 100 levels of brightness, which is controlled by the number 0 -99. These 100 levels of RGB colors combine up to $100 \times 100 \times 100$, or 1 million colors. At 0 , the lights are turn off and at 99, the lights are most bright. When the values of the three colors are the same, the light is white.

\subsection{Power Design}

A reliable power supply system is critical to the lighting control system, especially in the real industrial environment with complicated influence factors. So we design a reliable power supply system, table 1 introduces the power distribution of the main board in detail.

Table 1 Main board power distribution

\begin{tabular}{|c|c|c|c|c|}
\hline Type & Current & Ripple & Function & Source \\
\hline A-12V & $20.0 \mathrm{~A}$ & $80 \mathrm{mV}$ & For Lighting driver & INTPUT \\
\hline A-12V & $10.0 \mathrm{~A}$ & $80 \mathrm{mV}$ & For LDO & INTPUT \\
\hline C-5V & $2.0 \mathrm{~A}$ & $80 \mathrm{mV}$ & For LDO、CAN/485、AD & LDO \\
\hline A-3.3V & $800 \mathrm{~mA}$ & $50 \mathrm{mV}$ & For SCM & LDO \\
\hline
\end{tabular}

\section{System Software Design}

\subsection{Master control program design}


The STM32 master board control the light drive module in accordance with the instructions of the man-machine interface. When the system starts, the STM32 master board will check its own condition first. The range of inspection mainly includes the condition of light drive module, the status of the $12 \mathrm{~V}$ switching power supply for the LED and the state of communication between human-computer interaction interface and cameras. The specific process is shown in Fig.7.

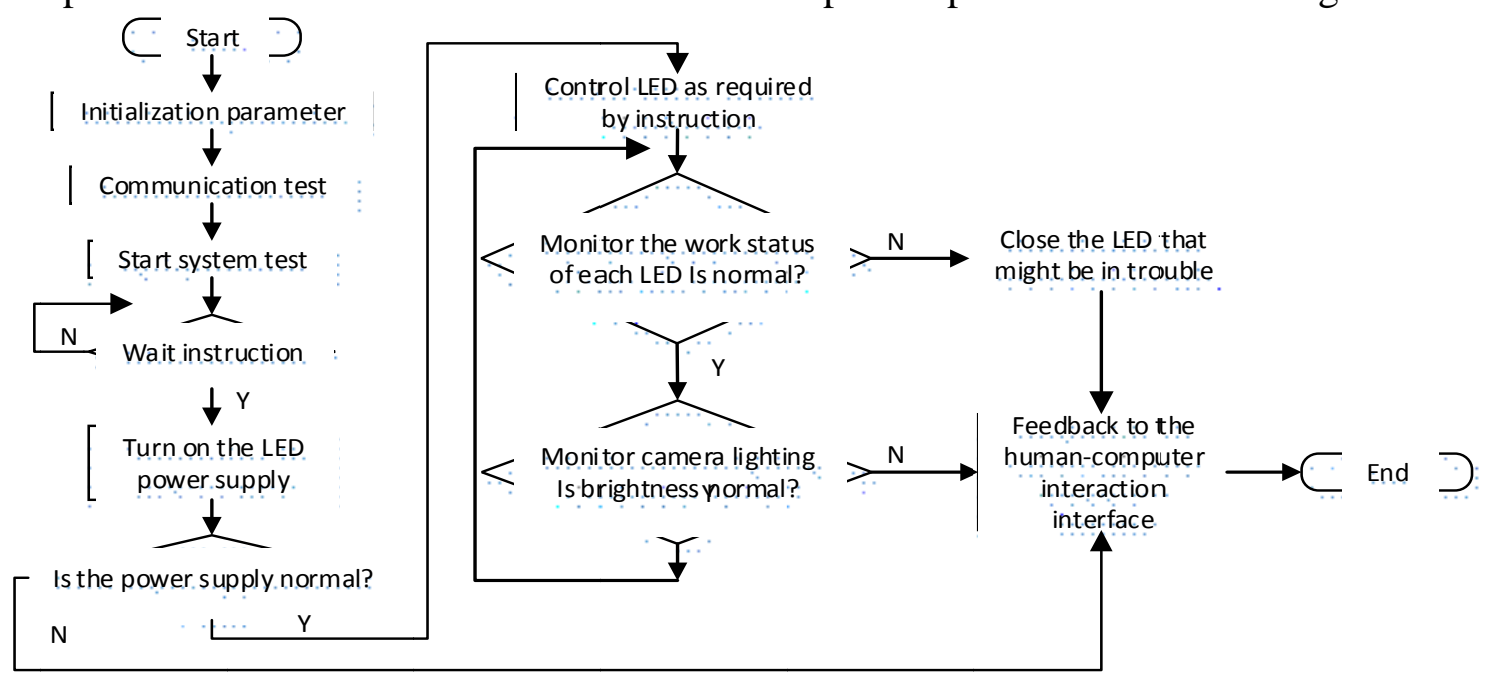

Fig.7 Main control program block diagram

\subsection{Upper computer program design}

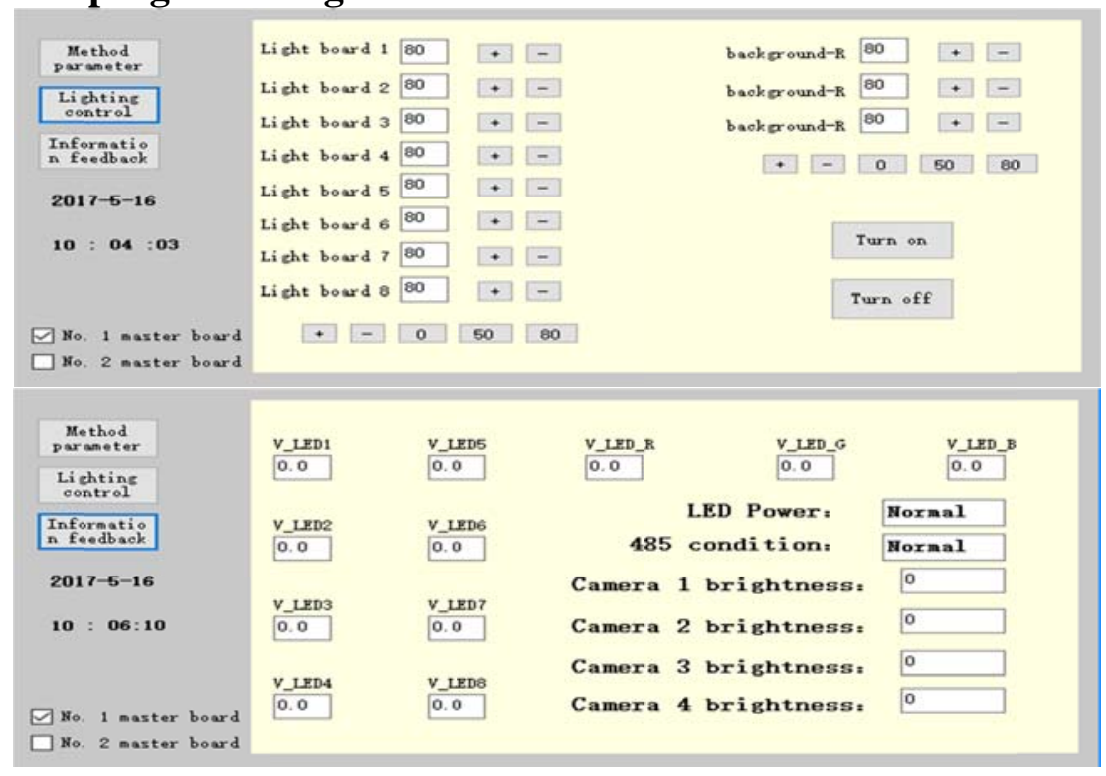

Fig.8 Human-computer interaction interface

The interface of the upper computer is shown in Fig.8, this interface can controls two STM32 master board by checking 1 and 2 to distinguish. Each STM32 master board is equipped with a dial switch and the ID of the master board is set by the dialing code switches. When you want to control a master board, the human-computer interaction interface will write the corresponding master board ID to the signal frame, such as table 2 and the master will only do so when reading the frame signal with its own ID. The interface can control the host side of the 8-way white LED lights and all the way RGB three-color background lights, brightness control range is 0 to 99 . The "+", "-" controls on the right side of each light panel indicate the addition and subtraction of the brightness of the light. The "Turn off" controls means to turn off all lights and the" Turn on" controls indicates that each light bar is lit in accordance with the data stored in the flash. Table 3 is the information feedback frame. 
Table 2 Light control signal frame

\begin{tabular}{|c|c|c|c|}
\hline Header & ID & Light 1 8+R G B & Sum check \\
\hline 0X4E & 1B & $11 \mathrm{~B}$ & $2 \mathrm{~B}$ \\
\hline
\end{tabular}

Table 3 Signal feedback frame

\begin{tabular}{|c|c|c|c|}
\hline Header & Light Voltage 1 8+R G B & Camera brightness & Sum check \\
\hline 0X4F & $11 \mathrm{~B}$ & $4 \mathrm{~B}$ & $2 \mathrm{~B}$ \\
\hline
\end{tabular}

\subsection{The design of communication program between cameras and the master board}

Here we let the main board connect with four cameras (or more) via the CAN bus. In the CAN bus, each node has its own ID, so it is easy to determine the order of transmission via the ID number and that communication will not be confused. What's more, the CAN communication protocol comes with CRC test, which can greatly improve the reliability of communication.

\section{Lighting Control System Test}

As we all know that a reliable control system must go through rigorous long-term、 multi-level industrial field test before normal use, especially as machine vision which is a high accuracy control system in industrial, it must want to ensure the safety and reliability of the various modules, so we have done the test.

(1) The test of power failure recovery. There may be a power failure in an actual working environment, so the system's self-recovery is very important. When we manually simulate power failure, the light control system can be restored the state of stored in the flash.

(2) The test of light brightness control of each road. When we input and change the brightness information of each light on the man-machine interface separately, the master board can control the brightness of the corresponding light by the information accurately.

(3) Abnormal condition feedback test. When we cut off the $12 \mathrm{~V}$ power supplied to the light driver, the man-machine interface shows "power supply abnormalities". In the system, the main control unit can monitor the working condition of each light panel, and on the feedback interface, the corresponding "V_LED" will be shown red. When the communication between the human-computer interface and the main control is disconnected, "communication abnormal" is displayed on the feedback interface.

(4) The communication test between the four camera and the main control unit. The test proves that the brightness of each camera can be displayed in real time on human-computer interaction interface, and the range of value is 0-255.

(5) Fig.9 is $10 \mathrm{khz}$ line frequency linear CCD camera collection DuPont line photos in the lighting control system. As you can see, the image is very clear.

(6) Table 4 shows the variation of the LED group voltage with PWM duty cycle, which is our actual measurement. Fig.10 is the relationship between the LED voltage and the PWM. It is shown by the graph that the two are approximately linear, so the system meets the LED brightness adjustment accuracy.

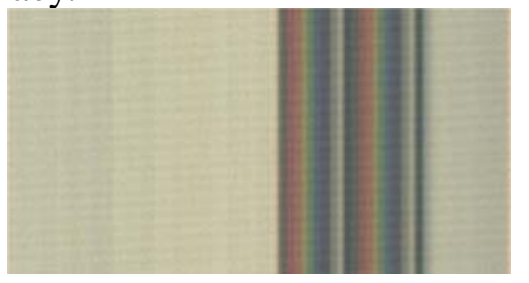

Fig.9 Collection of DuPont line photos 
Table 4 LED terminal voltage and corresponding PWM

\begin{tabular}{|c|c|c|c|c|c|c|c|}
\hline PWM \% & $0 \%$ & $5 \%$ & $10 \%$ & $15 \%$ & $20 \%$ & $25 \%$ & $30 \%$ \\
\hline V_LED /v & 5.89 & 6.87 & 6.97 & 7.09 & 7.20 & 7.33 & 7.46 \\
\hline PWM \% & $35 \%$ & $40 \%$ & $45 \%$ & $50 \%$ & $55 \%$ & $60 \%$ & $65 \%$ \\
\hline V_LED /v & 7.59 & 7.73 & 7.86 & 8.00 & 8.13 & 8.26 & 8.38 \\
\hline PWM \% & $70 \%$ & $75 \%$ & $80 \%$ & $85 \%$ & $90 \%$ & $95 \%$ & $100 \%$ \\
\hline V_LED /v & 8.50 & 8.62 & 8.73 & 8.84 & 9.02 & 9.20 & 9.33 \\
\hline
\end{tabular}

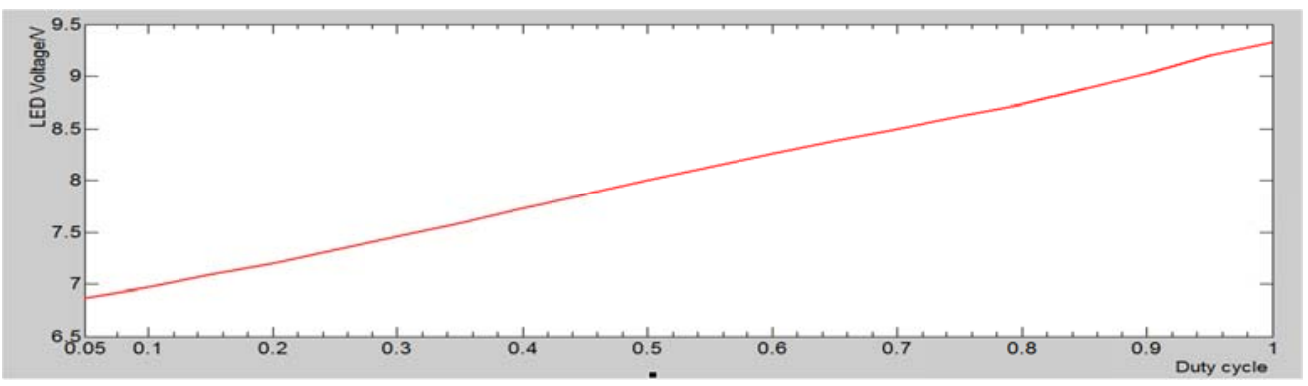

Fig.10 LED voltage fitting in MATLAB

\section{Conclusion}

The lighting control system completed in this paper can improve the quality of image acquisition. After a long period of field testing, the system can work stably from the man-machine interface to the host. The system can also feedback some error messages to improve the security and reliability of the whole system. Compared with the traditional lighting control system, the dimming accuracy of the system is higher, the quality of the light source is better, and because the design of man-machine interface makes the whole system more humane. It's all create better promotional value for the lighting control system.

\section{Acknowledgements}

This work was supported in part by National Natural Science Foundation of China (U1609216) and Public Projects of Zhejiang Province (2016C31069).

\section{References}

[1] SHIWAYA T Y,KISHIOKA T T,HAGINO K K. Power supply device and LED device and electronic device using same: USA,US 756419[P].2009-07-21.

[2] Li Ran Wu Junfeng Wang Haiying Design Method of CAN BUS Network Communication Structure for Electric Vehicle [C]/Strategic Technology(IFOST).2010 International Forum.2010:326-329

[3] Yan Zhang and Jinjuan Huang, “The research and application of intelligent watt-hour meter verification line system,"

Electrical Measurement \& Instrumentation, Vol.46, pp74-77, 2011

[4] D. Kim, E. Murphy-Hill, C. Parnin, C. Bird and R. Garcia, "The Reaction of Open-Source Projects to New Language Features: An Empirical Study of C\# Generics,” Journal of Object Technology, vol. 12, 2012 
[5] D. Kim, E. Murphy-Hill, C. Parnin, C. Bird and R. Garcia, "The Reaction of Open-Source Projects to New Language Features: An Empirical Study of C\# Generics,” Journal of Object Technology, vol. 12, 2012

[6] D. D. Damayanti, "Reconfigurable mixed model assembly line design in a dynamic production environment,” IEEE, pp568-572, 2012. 